\title{
Subspecialties in Neurosurgery: Are We Ready?
}

\author{
Dr Yam Bahadur Roka \\ MS, M.Ch., IFAANS
}

Nepalese Society of Neurosurgeons (NESON) opened a new episode in its history by forming the NeuroVascular chapter in April 2018. It was not the first though as a Spine chapter had already been formed two years ago. In as much as I would like to congratulate the new team I want to also discuss few things regarding neurosurgery subspecialties in Nepal.

With the advancement in medical sciences more and more departments have entered the phase where the work by one or few are limited to only aspect of the whole subject. In neurosurgery this could be Anatomicalspine, peripheral nerves or vascular, Disease orientatedVascular, trauma, tumor,infection etc., or age relatedpediatric, degenerative and could be functional like epilepsy, Parkinson's or other movement disorders. As rightly said "as the focus narrows, while the object in the center becomes visible with greater clarity, the surrounding areas become increasingly distorted". ${ }^{1}$ With this overlap of subspecialties are we ready in Nepal to start other chapters too? A neurosurgeon in the periphery working alone will have to address all and any type of neurosurgical problems he has training in, is capable of and he can manage. ${ }^{2}$ With the scarce neurosurgeons we have in Nepal we are yet to get proper referral of a disease to a specific subspecialtyneurosurgeon-as there is none yet.

A pediatric tumor or spine tumor, should it be managed by the pediatric or spine neurosurgeon respectively or the Neuro-oncosurgeon. Would a case of vascular malformation in a 16-year old be managed by the pediatric or the neurovascular surgeon? Training in subspecialty may be one of the factors to assess the outcome in treatment but there are many senior neurosurgeons with no such formal training yet are able to do complex neurosurgical procedures. So, should we go for the Degree or go for Experience. National training programs in neurosurgery was started in Nepal around 2000 and there is satisfactory number of neurosurgeons who qualify each year from four Medical Schools. ${ }^{3}$
Presently the new subspecialty chapters are holding regular CME, meetings, update and even international training/workshop for residents. Special sessions related to them are held in National and International NESON conferences. With the introduction of Functional Neurosurgery, Neurointervention and the availability of Neuronavigation, Modular operation theatre, standard instruments and latest generation of CT, PET, Radiosurgery and MRI scan in the country, neurosurgical progress has been rapid over the years. With the increasing number of neurosurgeons, the centers with neurosurgical facilities has increased all over the country. In future a time will come when the increased volume will mandate the formation of newer chapters in the society.

I would like to also give the example of the concept of "The Knowledge Doubling Time" of human knowledge which doubled in 100 years before 1900, took 25 years to double by World War II and at present takes only 18 months to double. ${ }^{4}$ Which means that any work done at present will be old or even obsolete within two years. In this scenario it will be pertinent that NESON too will have to develop more and more sub-specialties to cater to the increased number of neurosurgeons and to the advancements in neurosurgery. This will necessitate proper guidelines and protocol to develop centers where training can be done in the respective sub-specialties. ${ }^{5}$

Opening of such chapters thus is in my view the first step towards sub-specialization and I am sure NESON will as with other international societies make a niche in the neurosurgical world by their contribution. Although NESON with around 80 members looks small in comparison to neighboring India (1800 neurosurgeons) or USA \& Canada (4000 neurosurgeons), I am sure as time goes by we will be at one be at par with the neurosurgery in developed countries.1,6 NESON will have to follow guidelines from other societies regarding opening and training subspecialties and formulate their own rules based on the loco-regional geographic, ethnic, political and medical statistics. ${ }^{5-7}$ 


\section{References:}

1. Couldwell WT, Rovit RL. Rethinking neurosurgical subspecialization. Surg Neuro 158:359-63; discussion 363-70, 2002

2. Hughes M. Expanding nepalese neurosurgery: challenges and opportunities. World Neurosurg 73:436-7, 2010

3. Shilpakar SK. Subspecialties in neurosurgery and its challenges in a developing country. World Neurosurg 75:335-7, 2011

4. Schilling, D.R. The Knowledge Doubling Curve. Industry tap into the news. 2013
5. Frederick A. Boop. Subspecialty Training in Neurological Surgery. Inside Neurosurgeon | President's Perspective. AANS Neurosurgeon: 25:4, 2016

6. Ganapathy K. Neurosurgery in India: an overview. World Neurosurg 79:621-8, 2013

7. Piepgras DG. Post residency subspecialty training in neurosurgery--the impact of subspecialty training on organized neurosurgery and resident training-benefits, responsibilities and liabilities. Acta Neurochir Suppl 69:140-4, 1997 